\title{
On a class of semilinear fractional elliptic equations involving outside Dirac data
}

\author{
Huyuan Chen 1 Hichem Hajaiej 2 Ying Wang 3
}

\begin{abstract}
The purpose of this article is to give a complete study of the weak solutions of the fractional elliptic equation

$$
\begin{aligned}
(-\Delta)^{\alpha} u+u^{p}=0 & \text { in } \quad B_{1}\left(e_{N}\right), \\
u=\delta_{0} & \text { in } \quad \mathbb{R}^{N} \backslash B_{1}\left(e_{N}\right),
\end{aligned}
$$

where $p \geq 0,(-\Delta)^{\alpha}$ with $\alpha \in(0,1)$ denotes the fractional Laplacian operator in the principle value sense, $B_{1}\left(e_{N}\right)$ is the unit ball centered at $e_{N}=(0, \cdots, 0,1)$ in $\mathbb{R}^{N}$ with $N \geq 2$ and $\delta_{0}$ is the Dirac mass concentrated at the origin. We prove that problem (0.1) admits a unique weak solution when $p>1+\frac{2 \alpha}{N}$. Moreover, if in addition $p \geq \frac{N+2}{N-2}$, the weak solution vanishes as $\alpha \rightarrow 1^{-}$. We also show that problem (0.1) doesn't have any weak solution when $p \in\left[0,1+\frac{2 \alpha}{N}\right]$. These results are very surprising since there are in total contradiction with the classical setting, i.e.

$$
\begin{aligned}
& -\Delta u+u^{p}=0 \quad \text { in } B_{1}\left(e_{N}\right), \\
& u=\delta_{0} \quad \text { in } \mathbb{R}^{N} \backslash B_{1}\left(e_{N}\right),
\end{aligned}
$$

for which it has been proved that there are no solutions for $p \geq \frac{N+1}{N-1}$.

Key words: Fractional Laplacian; Dirac mass; Weak solution; Existence; Uniqueness.

MSC2010: 35R06, 35A01, 35J66.

\section{Introduction}

Fractional PDEs have gained tremendous interest, not only from mathematicians but also from physicists and engineering, during the last years. This is essentially due to their widespread domains of applications. In fact the fractional Laplacian arises in many areas including medicine [12], bioengineering [19, 20, 21, 22, relativistic physics[1, 17, 18, Modeling populations [29], flood flow, material viscoelastic theory, biology and earthquakes. It is also particularly relevant to study some situations, in which the fractional Laplacian is involved in PDEs, featuring irregular data such that those phenomena describing source terms which are concentrated at points. In our context, the source is placed outside the unit ball $B_{1}\left(e_{N}\right)$. This generates long-term interactions and shortterm interactions, described by the nonlocal operator $(-\Delta)^{\alpha}$ and the nonlinear absorption $u^{p}$ respectively. $(-\Delta)^{\alpha}$ has also a probabilistic interpretation, related to the above one. It is the $\alpha$-stable subordinated infinitesimal killed Brownian motion.

Let $B_{1}\left(e_{N}\right)$ be the unit ball in $\mathbb{R}^{N}(N \geq 2)$ with center $e_{N}=(0, \cdots, 0,1)$ and $\delta_{0}$ be the Dirac mass concentrated at the origin. Our main objective in this article is to investigate the existence, nonexistence and uniqueness of positive weak solutions of the semilinear fractional equation

$$
\begin{aligned}
(-\Delta)^{\alpha} u+u^{p}=0 & \text { in } \quad B_{1}\left(e_{N}\right), \\
u=\delta_{0} & \text { in } \quad \mathbb{R}^{N} \backslash B_{1}\left(e_{N}\right),
\end{aligned}
$$

where $p \geq 0$ and the fractional Laplacian $(-\Delta)^{\alpha}$ with $\alpha \in(0,1)$ is defined by

$$
(-\Delta)^{\alpha} u(x)=c_{N, \alpha} \lim _{\epsilon \rightarrow 0^{+}}(-\Delta)_{\epsilon}^{\alpha} u(x),
$$

\footnotetext{
${ }^{1}$ hc64@nyu.edu

${ }^{2}$ hh62@nyu.edu

3 yingwang00@126.com
} 
where

$$
c_{N, \alpha}=\left(\int_{\mathbb{R}^{N}} \frac{1-\cos \left(z_{1}\right)}{|z|^{N+2 \alpha}} d z\right)^{-1}
$$

with $z=\left(z_{1}, \cdots, z_{N}\right) \in \mathbb{R}^{N}$ and

$$
(-\Delta)_{\epsilon}^{\alpha} u(x)=-\int_{\mathbb{R}^{N} \backslash B_{\epsilon}(x)} \frac{u(z)-u(x)}{|z-x|^{N+2 \alpha}} d z .
$$

In 1991, a fundamental contribution to semilinear elliptic equations involving measures as boundary data is due to Gmira and Véron [15], where they studied the existence and uniqueness of weak solutions for

$$
\begin{aligned}
-\Delta u+h(u)=0 \quad & \text { in } \quad \Omega, \\
u=\mu & \text { on } \quad \partial \Omega,
\end{aligned}
$$

where $\Omega$ is a bounded $C^{2}$ domain and $\mu$ is a bounded Radon measure defined in $\partial \Omega$. A function $u$ is said to be a weak solution of (1.3) if $u \in L^{1}(\Omega), h(u) \in L^{1}(\Omega, \rho d x)$ and

$$
\int_{\Omega}[u(-\Delta) \xi+h(u) \xi] d x=\int_{\partial \Omega} \frac{\partial \xi(x)}{\partial \vec{n}_{x}} d \mu(x), \quad \forall \xi \in C_{0}^{1.1}(\Omega),
$$

where $\rho(x)=\operatorname{dist}(x, \partial \Omega)$ and $\vec{n}_{x}$ denotes the unit inward normal vector at a point $x$. Gmira and Véron proved that the problem (1.3) admits a unique weak solution when $h$ is a continuous and nondecreasing function satisfying

$$
\int_{1}^{\infty}[h(s)-h(-s)] s^{-1-\frac{N+1}{N-1}} d s<+\infty .
$$

The weak solution of (1.3) is approached by the classical solutions of (1.3) when $\mu$ is replaced by a sequence of regular functions $\left\{\mu_{n}\right\}$, which converge to $\mu$ in the distribution sense. Furthermore, they showed that there is no weak solution of (1.3) when $\mu=\delta_{x_{0}}$ with $x_{0} \in \partial \Omega$ and $h(s)=|s|^{p-1} s$ with $p \geq \frac{N+1}{N-1}$. Later on, this subject has been vastly expanded in recent works, see the papers of Marcus and Véron [23, 24, 25, 26], Bidaut-Véron and Vivier [3] and references therein.

In the fractional setting, the equivalent of (1.3) when $\mu=\delta_{x_{0}}$ has been considered in [7], where the authors proved that the weak solution of

$$
\begin{aligned}
& (-\Delta)^{\alpha} u+g(u)=k \frac{\partial^{\alpha} \delta_{x_{0}}}{\partial \vec{n}^{\alpha}} \quad \text { in } \quad \bar{\Omega}, \\
& u=0 \quad \text { in } \quad \bar{\Omega}^{c}
\end{aligned}
$$

is approximated by the weak solutions, as $t \rightarrow 0^{+}$, of

$$
\begin{aligned}
& (-\Delta)^{\alpha} u+g(u)=k t^{-\alpha} \delta_{x_{0}+t \vec{n}_{x_{0}}} \quad \text { in } \quad \bar{\Omega}, \\
& u=0 \quad \text { in } \quad \bar{\Omega}^{c} .
\end{aligned}
$$

More precisely, in the fractional setting, $\frac{\partial^{\alpha} \delta_{x_{0}}}{\partial \vec{n}^{\alpha}}$ plays the same role of $u=\delta_{x_{0}}$ on $\partial \Omega$ in (1.3). Our purpose in this article is to study the solution of

$$
(-\Delta)^{\alpha} u+u^{p}=0 \quad \text { in } \quad B_{1}\left(e_{N}\right)
$$

when the exact Dirac mass concentrated at the origin is considered. Our main idea is to make use of nonlocal properties of the fractional Laplacian to move the Dirac mass at $-t e_{N}$ when $t \rightarrow 0^{+}$ and we then proceed by approximation techniques. Before giving our main results, we must first give an appropriate definition of weak solution of (1.1). It is then worth to mention two important results. The equation

$$
\begin{aligned}
(-\Delta)^{\alpha} u+u^{p}=0 & \text { in } \quad B_{1}\left(e_{N}\right), \\
u=f & \text { in } \mathbb{R}^{N} \backslash B_{1}\left(e_{N}\right),
\end{aligned}
$$


where $f \in C_{0}\left(\mathbb{R}^{N} \backslash B_{1}\left(e_{N}\right)\right)$, admits a unique classical solution $u_{f}$, see [6, Theorem 2.5]. Furthermore, let $\tilde{u}_{f}=u_{f}$ in $B_{1}\left(e_{N}\right)$ and $\tilde{u}_{f}=0$ in $\mathbb{R}^{N} \backslash B_{1}\left(e_{N}\right)$, then $\tilde{u}_{f}$ is the unique classical solution of

$$
\begin{aligned}
(-\Delta)^{\alpha} u+u^{p} & =c_{N, \alpha} \int_{\mathbb{R}^{N} \backslash B_{1}\left(e_{N}\right)} \frac{f(y)}{|x-y|^{N+2 \alpha}} d y & & \text { in } B_{1}\left(e_{N}\right), \\
u & =0 & & \text { in } \mathbb{R}^{N} \backslash B_{1}\left(e_{N}\right)
\end{aligned}
$$

and satisfies the identity:

$$
\int_{B_{1}\left(e_{N}\right)}\left[u(x)(-\Delta)^{\alpha} \xi(x)+u^{p}(x) \xi(x)\right] d x=c_{N, \alpha} \int_{B_{1}\left(e_{N}\right)} \int_{\mathbb{R}^{N} \backslash B_{1}\left(e_{N}\right)} \frac{\xi(x) f(y)}{|x-y|^{N+2 \alpha}} d y d x
$$

for any $\xi \in C_{0}^{\infty}\left(B_{1}\left(e_{N}\right)\right)$. Let us mention that $C_{0}^{\infty}\left(B_{1}\left(e_{N}\right)\right)$ is the space of test functions $\xi \in$ $C^{\infty}\left(\mathbb{R}^{N}\right)$ with support in $B_{1}\left(e_{N}\right)$.

Inspired by above identity, we give the definition of weak solution to (1.1) as follows.

Definition 1.1 We say that $u$ is a weak solution of (1.1) if $u \in L^{1}\left(B_{1}\left(e_{N}\right)\right), u^{p} \in L_{\mathrm{loc}}^{1}\left(B_{1}\left(e_{N}\right)\right)$ and

$$
\int_{B_{1}\left(e_{N}\right)}\left[u(x)(-\Delta)^{\alpha} \xi(x)+u^{p}(x) \xi(x)\right] d x=\int_{B_{1}\left(e_{N}\right)} \xi(x) \Gamma_{0}(x) d x, \quad \forall \xi \in C_{0}^{\infty}\left(B_{1}\left(e_{N}\right)\right),
$$

where

$$
\Gamma_{0}(x)=\frac{c_{N, \alpha}}{|x|^{N+2 \alpha}}, \quad \forall x \in \mathbb{R}^{N} \backslash\{0\} .
$$

It is well known that the definition of the weak solution heavily depends on the test functions space and the best function space is the one which enables us to get the "strongest" weak solution. In [9, 10], semilinear fractional equations with measures has been studied via the test functions space $\mathbb{X}_{\alpha, \Omega} \subset C\left(\mathbb{R}^{N}\right)$ for a $C^{2}$ bounded open domain $\Omega$, where $\mathbb{X}_{\alpha, \Omega}$ is the space of functions $\xi$ satisfying:

(1) $\operatorname{supp}(\xi) \subset \bar{\Omega}$;

(2) $(-\Delta)^{\alpha} \xi(x)$ exists for all $x \in \Omega$ and $\left|(-\Delta)^{\alpha} \xi(x)\right| \leq C$ for some $C>0$;

(3) there exist $\varphi \in L^{1}\left(\Omega, \rho^{\alpha} d x\right)$ and $\epsilon_{0}>0$ such that $\left|(-\Delta)_{\epsilon}^{\alpha} \xi\right| \leq \varphi$ a.e. in $\Omega$, for all $\epsilon \in\left(0, \epsilon_{0}\right]$, where $\rho(x)=\operatorname{dist}(x, \partial \Omega)$.

The test functions space $C_{0}^{\infty}\left(B_{1}\left(e_{N}\right)\right)$ has stronger topology than $\mathbb{X}_{\alpha, B_{1}\left(e_{N}\right)}$ does, the weak solution

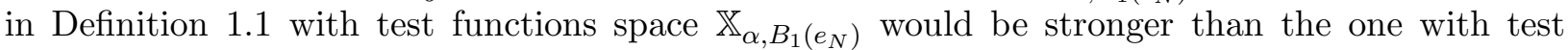
functions space $C_{0}^{\infty}\left(B_{1}\left(e_{N}\right)\right)$. It is then worth to mention that the test functions space $C_{0}^{\infty}\left(B_{1}\left(e_{N}\right)\right)$ could not be replaced to the test functions space $\mathbb{X}_{\alpha, B_{1}\left(e_{N}\right)}$ in our setting. For example, $\xi_{0}:=$ $\mathbb{G}_{\alpha}[1] \in \mathbb{X}_{\alpha, B_{1}\left(e_{N}\right)}$, but (1.9) does not hold for $\xi_{0}$, where $G_{\alpha}$ denotes the Green kernel of $(-\Delta)^{\alpha}$ in $B_{1}\left(e_{N}\right) \times B_{1}\left(e_{N}\right)$ and $\mathbb{G}_{\alpha}$ is the Green operator defined as

$$
\mathbb{G}_{\alpha}[f](x)=\int_{B_{1}\left(e_{N}\right)} G_{\alpha}(x, y) f(y) d y, \quad f \in L^{1}\left(B_{1}\left(e_{N}\right), \rho^{\alpha} d x\right) .
$$

Let us state our existence result.

Theorem 1.1 Assume that $\alpha \in(0,1)$ and $p>1+\frac{2 \alpha}{N}$. Then there exists a unique nonnegative weak solution $u_{\alpha, p}$ of (1.1) such that for some $c_{1}>1$, we have

$$
0<u_{\alpha, p}(x) \leq c_{1}|x|^{-\frac{N+2 \alpha}{p}}, \quad \forall x \in B_{1}\left(e_{N}\right)
$$

and

$$
\frac{1}{c_{1}} t^{-\frac{N+2 \alpha}{p}} \leq u_{\alpha, p}\left(t e_{N}\right) \leq c_{1} t^{-\frac{N+2 \alpha}{p}}, \quad \forall t \in(0,1) .
$$


Remark 1.1 (i) The existence results are very surprising as they are in total different from the Laplacian case, where (1.3) with $\mu=\delta_{0}$ has a weak solution only when $p<\frac{N+1}{N-1}$.

(ii) From (1.13), the singularity is only near the origin. We also notice that

$$
u_{\alpha, p}^{p}\left(t e_{N}\right) \geq \frac{1}{c_{1}} t^{-(N+2 \alpha)}, \quad \forall t \in(0,1),
$$

which implies that the absorption nonlinearity $u^{p}$ plays a primary role in the equation (1.1). While the absorption nonlinearity always plays a second role in a measure framework.

(iii) The uniqueness cannot directly follow Kato's inequality [9, Proposition 2.4] since it has been built in the framework of the test functions space $\mathbb{X}_{\alpha, B_{1}\left(e_{N}\right)}$. In this paper, as mentioned above, $C_{0}^{\infty}\left(B_{1}\left(e_{N}\right)\right)$ is the appropriate test functions space. This will give birth to a lot of technical difficulties to prove the existence, nonexistence and uniqueness of weak solutions of (1.1).

If $p>1+\frac{2 \alpha}{N}$, the weak solution $u_{\alpha, p}$ of (1.1) is approximated by the unique solution $u_{s}$ $(s \in(0,1))$ of

$$
\begin{aligned}
(-\Delta)^{\alpha} u+u^{p}=0 & \text { in } & B_{1}\left(e_{N}\right), \\
u=\delta_{-s e_{N}} & \text { in } & \mathbb{R}^{N} \backslash B_{1}\left(e_{N}\right) .
\end{aligned}
$$

When $p \in\left[0,1+\frac{2 \alpha}{N}\right]$, we will prove that $\left\{u_{s}\right\}$ blows up everywhere in $B_{1}\left(e_{N}\right)$ as $s \rightarrow 0^{+}$, therefore, we can deduce the nonexistence of weak solutions of (1.1) when $p \leq 1+\frac{2 \alpha}{N}$. More precisely, we have the following results.

Theorem 1.2 Assume that $\alpha \in(0,1)$ and $0 \leq p \leq 1+\frac{2 \alpha}{N}$. Then problem (1.1) does not have any weak solution.

In the proof of Theorem 1.2, we will first need to prove the crucial estimate

$$
u_{\alpha, p}^{p}(x) \geq c_{2}|x|^{-(N+2 \alpha)}, \quad \forall x \in \mathcal{C},
$$

where $\mathcal{C}=\left\{x \in \mathbb{R}^{N}: \exists t \in(0,1)\right.$ s.t. $\left.\left|x-t e_{N}\right|<\frac{t}{8}\right\}$ is a cone in $B_{1}\left(e_{N}\right)$. We combine the symmetry property and decreasing monotonicity in our proof of the nonexistence. This phenomena is due to the nonlocal characteristic of fractional Laplacian that requires the functions to be in $L_{\text {loc }}^{1}\left(\mathbb{R}^{N}\right)$.

Finally, our interest is to study the asymptotic behavior of $u_{\alpha, p}$ as $\alpha$ goes to $1^{-}$.

Theorem 1.3 Assume that $\alpha \in(0,1), p \geq \frac{N+1}{N-1}$ and $u_{\alpha, p}$ is the unique weak solution of problem (1.1). Then $\left\{u_{\alpha, p}\right\}_{\alpha}$ vanishes as $\alpha \rightarrow 1^{-}$.

Remark 1.2 (i) for $p \geq \frac{N+2}{N-2}$, a sequence of barrier functions, which converge to 0 locally in $B_{1}(0)$, could be constructed directly to control $\left\{u_{\alpha, p}\right\}_{\alpha}$;

(ii) for $\frac{N+1}{N-1} \leq p<\frac{N+2}{N-2}$, Theorem 3.1 in [15] is involved to control $\left\{u_{\alpha, p}\right\}_{\alpha}$;

(iii) for $p<1+\frac{2}{N}$, there exists $\alpha_{p} \in(0,1)$ such that $p \leq 1+\frac{2 \alpha}{N}$ for $\alpha \in\left(\alpha_{p}, 1\right)$, there is no weak solution for problem (1.1) from Theorem 1.2;

(iv) for $p \in\left[1+\frac{2}{N}, \frac{N+1}{N-1}\right)$, it is still open for the limit of $\left\{u_{\alpha, p}\right\}_{\alpha}$ as $\alpha \rightarrow 1^{-}$.

In Section 2, we treat the problem (1.14). When the Dirac mass concentrates at point $-s e_{N}$ away from $\bar{\Omega}$, we build the existence, uniqueness weak solution $u_{s}$ of (1.14) and show how the Dirac mass is transformed into the nonhomogeneous term. In this case, the test functions space could be improved into $\mathbb{X}_{\alpha, B_{1}\left(e_{N}\right)}$, since the solution has no singularity in $\bar{\Omega}$.

In Section 3, we give a detailed account of the procedure enabling us to move the singular points $\left\{-s e_{N}\right\}$ to the origin. The first difficulty arises from the fact that $\mathbb{G}_{\alpha}\left[\Gamma_{s}\right]$ blows up everywhere as $s \rightarrow 0^{+}$[see Lemma 3.1], that is, there is no solution of

$$
\begin{array}{rlll}
(-\Delta)^{\alpha} u=0 & \text { in } & B_{1}\left(e_{N}\right), \\
u=\delta_{0} & \text { in } & \mathbb{R}^{N} \backslash B_{1}\left(e_{N}\right) .
\end{array}
$$


Therefore, we have to resort a barrier function, that is the minimal classical solution of

$$
\begin{aligned}
(-\Delta)^{\alpha} u+u^{p}=\Gamma_{0} & & \text { in } & B_{1}\left(e_{N}\right), \\
u=0 & & \text { in } & B_{1}^{c}\left(e_{N}\right) \backslash\{0\} .
\end{aligned}
$$

In order to control the limit of $\left\{u_{s}\right\}$ near $\partial B_{1}$, especially near the origin, some typical truncated functions have to be constructed carefully. The second difficulty comes from the proof of the uniqueness. We proceed by contradiction, assuming that there is two solutions and we will show their difference could be improved the test function from $C_{0}^{\infty}$ into $\mathbb{X}_{\alpha, B_{1}\left(e_{N}\right)}$, this enables us to use Kato's inequality [9, Proposition 2.4] and to conclude.

Section 4 is devoted to blow-up case. The difficulty is to obtain the blow-up everywhere in $B_{1}\left(e_{N}\right)$ just from a lower bounds of $u_{s}$, see Lemma 4.1. To overcome it, we combine the symmetric of the domain and resort the symmetry result of $u_{s}$ and then one point blowing up leads to blowing up every where in $B_{1}\left(e_{N}\right)$.

Finally, we analyse decay approximation of the weak solution for problem (1.1) when $\alpha \rightarrow 1^{-}$. For $p \geq \frac{N+2}{N-2}$, the first challenge is to construct a sequence upper bounds that converges to zero. To this end, we have to study $\lim _{\alpha \rightarrow 1^{-}}(-\Delta)^{\alpha} \Phi_{\sigma}$, where $\Phi_{\sigma}(x)=|x|^{-\sigma}$ and then use proper parameters to construct the bounds. For $\frac{N+1}{N-1} \leq p \leq \frac{N+2}{N-2}, \Phi_{\sigma}$ could not be used to construct properly the upper bounds, and then we use some argument of [15].

\section{Dirac mass concentrated at $\left\{-s e_{N}\right\}$ with $s \in(0,1)$}

The purpose of this section is to introduce some preliminaries. First let us state an important Comparison Principle.

Theorem 2.1 [6, Theorem 2.3] Let $u$ and $v$ be super-solution and sub-solution, respectively, of

$$
(-\Delta)^{\alpha} u+h(u)=f \text { in } \mathcal{O},
$$

where $\mathcal{O}$ is an open, bounded and connected domain of class $C^{2}$, the function $f: \mathcal{O} \rightarrow \mathbb{R}$ is continuous and $h: \mathbb{R} \rightarrow \mathbb{R}$ is increasing.

Suppose that $v(x) \leq u(x), \forall x \in \mathcal{O}^{c}, u$ and $v$ are continuous in $\overline{\mathcal{O}}$. Then

$$
u(x) \geq v(x), \quad \forall x \in \mathcal{O} .
$$

Now we investigate the weak solution of

$$
\begin{aligned}
& (-\Delta)^{\alpha} u+u^{p}=0 \quad \text { in } \quad B_{1}\left(e_{N}\right), \\
& u=\delta_{-s e_{N}} \quad \text { in } \quad \mathbb{R}^{N} \backslash B_{1}\left(e_{N}\right),
\end{aligned}
$$

where $s \in(0,1)$. To this end, we construct a sequence of $C^{2}$ functions to approximate the Dirac measure. Let $g_{0}: \mathbb{R}^{N} \rightarrow[0,1]$ be a radially symmetric decreasing $C^{2}$ function with the support in $\overline{B_{\frac{1}{2}}(0)}$ such that $\int_{\mathbb{R}^{N}} g_{0}(x) d x=1$. For any $n \in \mathbb{N}$ and $s \in(0,1)$, we denote

$$
g_{n}(x)=n^{N} g_{0}\left(n\left(x+s e_{N}\right)\right), \quad \forall x \in \mathbb{R}^{N} .
$$

Then we certainly have that

$$
g_{n} \rightarrow \delta_{-s e_{N}} \text { as } n \rightarrow+\infty,
$$

in the distribution sense and for any $s>0$, there exists $N_{s}>0$ such that for any $n \geq N_{s}$,

$$
\operatorname{supp}\left(g_{n}\right) \subset \overline{B_{\frac{s}{2}}\left(-s e_{N}\right)} .
$$

In order to investigate the solution of (2.1), we consider the approximating solution $w_{n}$ of

$$
\begin{array}{rll}
(-\Delta)^{\alpha} u+u^{p}=0 & \text { in } & B_{1}\left(e_{N}\right), \\
u=g_{n} & \text { in } & \mathbb{R}^{N} \backslash B_{1}\left(e_{N}\right) .
\end{array}
$$


Lemma 2.1 Assume that $p>0$ and $\left\{g_{n}\right\}$ is a sequence of $C^{2}$ functions converging to $\delta_{-s e_{N}}$ with supports in $\overline{B_{\frac{s}{2}}\left(-s e_{N}\right)}$. Denote that

$$
\tilde{g}_{n}(x):=c_{N, \alpha} \int_{\mathbb{R}^{N}} \frac{g_{n}(y)}{|x-y|^{N+2 \alpha}} d y, \quad \forall x \in B_{1}\left(e_{N}\right) .
$$

Then problem (2.2) admits a unique solution $w_{n}$ such that

$$
0<w_{n} \leq \mathbb{G}_{\alpha}\left[\tilde{g}_{n}\right] \text { in } B_{1}\left(e_{N}\right) .
$$

Moreover, the function $\tilde{w}_{n}:=w_{n} \chi_{B_{1}\left(e_{N}\right)}$ is the unique solution of

$$
\begin{array}{rlll}
(-\Delta)^{\alpha} u+u^{p}=\tilde{g}_{n} & \text { in } & B_{1}\left(e_{N}\right), \\
u=0 & \text { in } & \mathbb{R}^{N} \backslash B_{1}\left(e_{N}\right) .
\end{array}
$$

Proof. The existence and uniqueness of solution to problem (2.2) refers to [6, Theorem 2.5]. For $n \geq N_{s}$, we have that $\operatorname{supp}\left(g_{n}\right) \subset B_{\frac{s}{2}}\left(-s e_{N}\right)$ and then $\tilde{g}_{n} \in C^{1}\left(\overline{B_{1}\left(e_{N}\right)}\right)$ and

$$
\tilde{w}_{n}=w_{n}-g_{n} \quad \text { in } \quad \mathbb{R}^{N} \text {. }
$$

By the definition of fractional Laplacian, it implies that

$$
\begin{aligned}
(-\Delta)^{\alpha} \tilde{w}_{n}(x)+\tilde{w}_{n}(x)^{p} & =(-\Delta)^{\alpha} w_{n}(x)-(-\Delta)^{\alpha} g_{n}(x)+w_{n}(x)^{p} \\
& =c_{N, \alpha} \int_{\mathbb{R}^{N}} \frac{g_{n}(z)}{|z-x|^{N+2 \alpha}} d z=\tilde{g}_{n}(x) .
\end{aligned}
$$

Therefore, $\tilde{w}_{n}$ is a classical solution of (2.4) and

$$
\tilde{w}_{n} \leq \mathbb{G}_{\alpha}\left[\tilde{g}_{n}\right] \text { in } B_{1}\left(e_{N}\right),
$$

which implies that

$$
w_{n} \leq \mathbb{G}_{\alpha}\left[\tilde{g}_{n}\right] \text { in } B_{1}\left(e_{N}\right)
$$

The proof ends.

We remark that $\tilde{w}_{n}$ is the classical solution of (2.4), then by Lemma 2.1 and Lemma 2.2 in [9], we have that

$$
\int_{B_{1}\left(e_{N}\right)}\left[w_{n}(-\Delta)^{\alpha} \xi+w_{n}^{p} \xi\right] d x=\int_{B_{1}\left(e_{N}\right)} \xi \tilde{g}_{n} d x, \quad \forall \xi \in C_{0}^{\infty}\left(B_{1}\left(e_{N}\right)\right) .
$$

Here (2.5) holds even for $\xi \in \mathbb{X}_{\alpha, B_{1}\left(e_{N}\right)}$.

Lemma 2.2 Let $\left\{\tilde{g}_{n}\right\}$ be defined in (2.3), then $\tilde{g}_{n}$ converges to $\Gamma_{s}$ uniformly in $B_{1}\left(e_{N}\right)$ and in $C^{\theta}\left(B_{1}\left(e_{N}\right)\right)$ for $\theta \in(0,1)$, where

$$
\Gamma_{s}(x)=\frac{c_{N, \alpha}}{\left|x+s e_{N}\right|^{N+2 \alpha}}, \quad \forall x \in \mathbb{R}^{N} \backslash\left\{-s e_{N}\right\} .
$$

Proof. It is obvious that $\tilde{g}_{n}$ converges to $\Gamma_{s}$ every point in $\overline{B_{1}\left(e_{N}\right)}$. For $x, y \in B_{1}\left(e_{N}\right)$ and any $n \in \mathbb{N}$, we have that

$$
\begin{aligned}
\left|\tilde{g}_{n}(x)-\tilde{g}_{n}(y)\right| & =c_{N, \alpha}\left|\int_{B_{\frac{s}{2}}\left(-s e_{N}\right)}\left[\frac{1}{|x-z|^{N+2 \alpha}}-\frac{1}{|y-z|^{N+2 \alpha}}\right] g_{n}(z) d z\right| \\
& \leq c_{N, \alpha} \int_{B_{\frac{s}{2}}\left(-s e_{N}\right)} \frac{|| x-\left.z\right|^{N+2 \alpha}-|y-z|^{N+2 \alpha} \mid}{|x-z|^{N+2 \alpha}|y-z|^{N+2 \alpha}} g_{n}(z) d z \\
& \leq c_{N, \alpha}(N+2 \alpha)|x-y| \int_{B_{\frac{s}{2}}\left(-s e_{N}\right)} \frac{|x-z|^{N+2 \alpha-1}+|y-z|^{N+2 \alpha-1}}{|x-z|^{N+2 \alpha}|y-z|^{N+2 \alpha}} g_{n}(z) d z \\
& \leq c_{3}|x-y| \int_{B_{\frac{s}{2}}\left(-s e_{N}\right)} g_{n}(z) d z \\
& =c_{3}|x-y|,
\end{aligned}
$$


where $c_{3}>0$ independent of $n$. So $\left\{\tilde{g}_{n}\right\}_{n}$ is uniformly bounded in $C^{0,1}\left(B_{1}\left(e_{N}\right)\right)$. Combining the converging

$$
\tilde{g}_{n} \rightarrow \Gamma_{s} \text { every point in } \overline{B_{1}\left(e_{N}\right)} .
$$

We conclude that $\tilde{g}_{n}$ converges to $\Gamma_{s}$ uniformly in $B_{1}\left(e_{N}\right)$ and in $C^{\theta}\left(B_{1}\left(e_{N}\right)\right)$ for $\theta \in(0,1)$.

Proposition 2.1 Assume that $p>0, s \in(0,1)$ and $\Gamma_{s}$ is given by (2.6). Then problem (2.1) admits a unique weak solution $u_{s}$ such that

$$
0 \leq u_{s}(x) \leq \mathbb{G}_{\alpha}\left[\Gamma_{s}\right], \quad x \in B_{1}\left(e_{N}\right) .
$$

Moreover, $\tilde{u}_{s}:=u_{s} \chi_{B_{1}\left(e_{N}\right)}$ is the unique classical solution of

$$
\begin{aligned}
(-\Delta)^{\alpha} u+u^{p}=\Gamma_{s} & \text { in } & B_{1}\left(e_{N}\right), \\
u=0 & \text { in } & \mathbb{R}^{N} \backslash B_{1}\left(e_{N}\right) .
\end{aligned}
$$

Proof. Existence. It infers by Lemma 2.1 that the solution $w_{n}$ of (2.2) satisfies that

$$
0<w_{n} \leq \mathbb{G}_{\alpha}\left[\tilde{g}_{n}\right] \quad \text { in } \quad B_{1}\left(e_{N}\right) .
$$

By Lemma 2.2 we have that $\tilde{g}_{n}$ converges to $\Gamma_{s}$ uniformly in $\overline{B_{1}\left(e_{N}\right)}$ and in $C^{\theta}\left(B_{1}\left(e_{N}\right)\right)$ with $\theta \in(0,1)$. Therefore, there exists some constant $c_{4}>0$ independent of $n$ such that

$$
\mathbb{G}_{\alpha}\left[\tilde{g}_{n}\right](x) \leq \frac{c_{4} c_{N, \alpha}}{\left|x+s e_{N}\right|^{N+2 \alpha}} \leq c_{4} c_{N, \alpha} s^{-N-2 \alpha}, \quad \forall x \in B_{1}\left(e_{N}\right)
$$

Thus,

$$
\left\|w_{n}\right\|_{L^{\infty}\left(B_{1}\left(e_{N}\right)\right)} \leq c_{4} c_{N, \alpha} s^{-N-2 \alpha}, \quad\left\|w_{n}\right\|_{L^{1}\left(B_{1}\left(e_{N}\right)\right)} \leq c_{4} c_{N, \alpha} s^{-N-2 \alpha}\left|B_{1}\left(e_{N}\right)\right| .
$$

By [28, Theorem 1.2], we have that

$$
\begin{aligned}
\left\|\frac{w_{n}}{\rho^{\alpha}}\right\|_{C^{\alpha}\left(\overline{B_{1}\left(e_{N}\right)}\right)} & \leq c_{5}\left[\left\|w_{n}^{p}\right\|_{L^{\infty}\left(B_{1}\left(e_{N}\right)\right)}+\left\|\Gamma_{s}\right\|_{L^{\infty}\left(B_{1}\left(e_{N}\right)\right)}\right] \\
& \leq c_{6}\left[s^{-N-2 \alpha}+s^{-(N+2 \alpha) p}\right]
\end{aligned}
$$

for some $c_{5}, c_{6}>0$.

In order to see the inner regularity, we denote $\mathcal{O}_{i}$ the open sets with $i=1,2,3$ such that

$$
\mathcal{O}_{1} \subset \overline{\mathcal{O}}_{1} \subset \mathcal{O}_{2} \subset \overline{\mathcal{O}}_{2} \subset \mathcal{O}_{3} \subset \overline{\mathcal{O}}_{3} \subset B_{1}\left(e_{N}\right) \text {. }
$$

By [11, Lemma 3.1], for $\beta \in(0, \alpha)$, there exists $c_{7}, c_{8}>0$ independent of $n$ such that

$$
\begin{aligned}
\left\|w_{n}\right\|_{C^{\beta}\left(\mathcal{O}_{2}\right)} & \leq c_{7}\left[\left\|w_{n}\right\|_{L^{1}\left(B_{1}\left(e_{N}\right)\right)}+\left\|w_{n}^{p}\right\|_{L^{\infty}\left(\mathcal{O}_{3}\right)}+\left\|w_{n}\right\|_{L^{\infty}\left(\mathcal{O}_{3}\right)}\right] \\
& \leq c_{8}\left[s^{-N-2 \alpha}+s^{-(N+2 \alpha) p}\right] .
\end{aligned}
$$

It follows by [28, Corollary 2.4] that there exist $c_{9}, c_{10}>0$ such that

$$
\begin{aligned}
\left\|w_{n}\right\|_{C^{2 \alpha+\beta}\left(\mathcal{O}_{1}\right)} & \leq c_{9}\left[\left\|w_{n}\right\|_{L^{1}\left(B_{1}\left(e_{N}\right)\right)}+\left\|w_{n}^{p}\right\|_{C^{\beta}\left(\mathcal{O}_{2}\right)}+\left\|w_{n}\right\|_{C^{\beta}\left(\mathcal{O}_{2}\right)}\right] \\
& \leq c_{10}\left[s^{-N-2 \alpha}+s^{-(N+2 \alpha) p}\right] .
\end{aligned}
$$

Therefore, by the Arzela-Ascoli Theorem, there exist $u_{s} \in C_{\text {loc }}^{2 \alpha+\epsilon}$ in $B_{1}\left(e_{N}\right)$ for some $\epsilon \in(0, \beta)$ and a subsequence $\left\{w_{n_{k}}\right\}$ such that

$$
w_{n_{k}} \rightarrow u_{s} \text { locally in } C^{2 \alpha+\epsilon} \quad \text { as } \quad n_{k} \rightarrow \infty .
$$


Passing the limit of (2.5) with $\xi \in \mathbb{X}_{\alpha, B_{1}\left(e_{N}\right)}$ as $n_{k} \rightarrow \infty$, we have that

$$
\int_{B_{1}\left(e_{N}\right)}\left[u_{s}(-\Delta)^{\alpha} \xi+u_{s}^{p} \xi\right] d x=\int_{B_{1}\left(e_{N}\right)} \xi(x) \Gamma_{s}(x) .
$$

Moreover, since $w_{n} \rightarrow u_{s}$ and $\tilde{g}_{n} \rightarrow \Gamma_{s}$ uniformly in $B_{1}\left(e_{N}\right)$ as $n \rightarrow \infty$, then it infers that

$$
0 \leq u_{s} \leq \mathbb{G}_{\alpha}\left[\Gamma_{s}\right] \quad \text { in } \quad B_{1}\left(e_{N}\right)
$$

Uniqueness. Let $v_{s}$ be a weak solution of (2.2) and then $\varphi_{s}:=u_{s}-v_{s}$ is a weak solution to

$$
\begin{array}{rlll}
(-\Delta)^{\alpha} \varphi_{s}+u_{s}^{p}-v_{s}^{p}=0 & \text { in } & B_{1}\left(e_{N}\right) \\
\varphi_{s}=0 & \text { in } & \mathbb{R}^{N} \backslash B_{1}\left(e_{N}\right) .
\end{array}
$$

By Kato's inequality [9, Proposition 2.4],

$$
\int_{B_{1}\left(e_{N}\right)}\left|\varphi_{s}\right|(-\Delta)^{\alpha} \xi+\int_{B_{1}\left(e_{N}\right)}\left[u_{s}^{p}-v_{s}^{p}\right] \operatorname{sign}\left(u_{s}-v_{s}\right) \xi d x=0 .
$$

Taking $\xi=\mathbb{G}_{\alpha}[1]$, we have that

$$
\int_{B_{1}\left(e_{N}\right)}\left[u_{s}^{p}-v_{s}^{p}\right] \operatorname{sign}\left(u_{s}-v_{s}\right) \xi d x \geq 0 \quad \text { and } \quad \int_{B_{1}\left(e_{N}\right)}\left|\varphi_{s}\right| d x=0
$$

then $\varphi_{s}=0$ a.e. in $B_{1}\left(e_{N}\right)$. Then the uniqueness is proved.

Furthermore, we see that $\tilde{w}_{n}=w_{n}-g_{n}$ is the unique classical solution of

$$
\begin{aligned}
(-\Delta)^{\alpha} u(x)+u^{p}(x) & =\tilde{g}_{n}(x), & & \forall x \in B_{1}\left(e_{N}\right), \\
u(x) & =0, & & \forall x \in B_{1}\left(e_{N}\right)^{c}
\end{aligned}
$$

and $\tilde{w}_{n}$ converges to $\tilde{u}_{s}$ uniformly in $B_{1}\left(e_{N}\right)$. By Stability Theorem [5, Lemma 4.5] and (2.12), $u_{s} \chi_{B_{1}\left(e_{N}\right)}$ is the classical solution of (2.8).

\section{Proof of Theorem 1.1.}

In this section, we prove Theorem 1.1 by moving the points $\left\{-s e_{N}\right\}$ to the origin. To this end, we need derive more properties for $u_{s}$, where $u_{s}$ is the unique weak solution of (2.1).

Lemma 3.1 Let $p>0, s \in(0,1)$ and $u_{s}$ be the unique weak solution of (2.1). Then the mapping $s \mapsto u_{s}$ is decreasing, that is,

$$
u_{s_{1}} \geq u_{s_{2}} \text { if } s_{1} \leq s_{2}
$$

Proof. By Proposition 2.1, $\tilde{u}_{s}:=u_{s} \chi_{B_{1}\left(e_{N}\right)}$ is the unique classical solution of (2.8).

We claim that the mapping: $s \mapsto \Gamma_{s}$ is decreasing. For $x \in B_{1}\left(e_{N}\right)$ and $s_{1} \leq s_{2}$, we observe that $\left|x+s_{1} e_{N}\right| \leq\left|x+s_{2} e_{N}\right|$, then $\Gamma_{s_{1}}(x) \geq \Gamma_{s_{2}}(x)$. The claim is proved.

Therefore, for $s_{1} \leq s_{2}, u_{s_{1}}$ and $u_{s_{2}}$ are super solution and solution of (2.8) replaced $\Gamma_{s}$ by $\Gamma_{s_{2}}$, then it infers by the Comparison Principle that $u_{s_{1}} \geq u_{s_{2}}$ in $B_{1}\left(e_{N}\right)$.

Lemma 3.2 Let $s \in(0,1)$ and denote

$$
\mathbb{G}_{\alpha}\left[\Gamma_{s}\right](x)=\int_{B_{1}\left(e_{N}\right)} G_{\alpha}(x, y) \Gamma_{s}(y) d y, \quad \forall x \in B_{1}\left(e_{N}\right) .
$$

Then

$$
\lim _{s \rightarrow 0^{+}} \mathbb{G}_{\alpha}\left[\Gamma_{s}\right](x)=+\infty, \quad \forall x \in B_{1}\left(e_{N}\right) .
$$


Proof. Using [8, Theorem 1.2], it follows that

$$
G_{\alpha}(x, y) \geq \min \left\{\frac{c_{11}}{|x-y|^{N-2 \alpha}}, \frac{c_{11} \rho^{\alpha}(x) \rho^{\alpha}(y)}{|x-y|^{N}}\right\}, \quad x, y \in B_{1}\left(e_{N}\right)
$$

where $c_{11}>0$ dependent of $N$, $\alpha$. Now for $x \in B_{1}\left(e_{N}\right)$ and $y \in B_{1}\left(e_{N}\right) \cap B_{\frac{|x|}{4}}(0)$, we have that

$$
G_{\alpha}(x, y) \geq \frac{c_{11} \rho^{\alpha}(x) \rho^{\alpha}(y)}{|x-y|^{N}}
$$

and

$$
\begin{aligned}
\mathbb{G}_{\alpha}\left[\Gamma_{s}\right](x) & \geq \int_{B_{1}\left(e_{N}\right)} \min \left\{\frac{c_{11}}{|x-y|^{N-2 \alpha}}, \frac{c_{11} \rho^{\alpha}(x) \rho^{\alpha}(y)}{|x-y|^{N}}\right\} \frac{c_{N, \alpha}}{\left|y+s e_{N}\right|^{N+2 \alpha}} d y \\
& \geq \int_{B_{1}\left(e_{N}\right) \cap B_{\frac{|x|}{4}}(0)} \frac{c_{11} \rho^{\alpha}(x) \rho^{\alpha}(y)}{|x-y|^{N}} \frac{c_{N, \alpha}}{\left|y+s e_{N}\right|^{N+2 \alpha}} d y \\
& \geq \frac{4}{5} c_{11} \rho^{\alpha}(x)|x|^{-N} \int_{B_{1}\left(e_{N}\right) \cap B_{\frac{|x|}{4}}(0)} \frac{c_{N, \alpha} \rho^{\alpha}(y)}{\left|y+s e_{N}\right|^{N+2 \alpha}} d y \\
& \rightarrow+\infty \quad \text { as } \quad s \rightarrow 0^{+} .
\end{aligned}
$$

The proof ends.

From Lemma 3.2, it is informed that the limit of $\mathbb{G}_{\alpha}\left[\Gamma_{s}\right]$ as $s \rightarrow 0^{+}$can't be used as a barrier function to control the sequence $\left\{u_{s}\right\}$. So we have to find new upper bound for sequence $\left\{u_{s}\right\}$.

Proposition 3.1 Let $\Gamma_{0}$ be defined in (1.10) and

$$
p>1+\frac{2 \alpha}{N},
$$

then problem

$$
\begin{aligned}
(-\Delta)^{\alpha} u+u^{p}=\Gamma_{0} & \text { in } \quad B_{1}\left(e_{N}\right), \\
u=0 & \text { in } \quad B_{1}^{c}\left(e_{N}\right) \backslash\{0\} .
\end{aligned}
$$

admits a minimum positive solution $u_{0}$, that is, $u_{0} \leq u$ for any nonnegative solution $u$ of (3.2).

Moreover,

$$
\lim _{x \in B_{1}\left(e_{N}\right), x \rightarrow \partial B_{1}\left(e_{N}\right) \backslash\{0\}} u_{0}(x)=0
$$

and

$$
\frac{1}{c_{12}} t^{-\frac{N+2 \alpha}{p}} \leq u_{0}\left(t e_{N}\right) \leq c_{12} t^{-\frac{N+2 \alpha}{p}}, \quad t \in(0,1)
$$

where $c_{12}>1$ is independent of $\alpha$.

Proof. The existence of solution to (3.2). It implies by Lemma 3.2 that the mapping $s \mapsto u_{s}$ is decreasing in $B_{1}\left(e_{N}\right)$, where $u_{s} \chi_{B_{1}\left(e_{N}\right)}$ is the solution of (2.8). So what we have to do is just to find a super solution $U$ of (3.2) such that $u_{0} \leq U$ in $B_{1}\left(e_{N}\right)$. To this end, we consider the radial function

$$
\Phi_{\sigma}(x)=\frac{1}{|x|^{\sigma}}, \quad \forall x \in \mathbb{R}^{N} \backslash\{0\},
$$

where $\sigma \in[0, N)$. By scaling property of $\Phi_{\sigma}$, (also see [13]) we know that

$$
(-\Delta)^{\alpha} \Phi_{\sigma}(x)=\frac{c(\sigma, \alpha)}{|x|^{\sigma+2 \alpha}}
$$


where $c(\sigma, \alpha) \in \mathbb{R}$. Now we choose $\sigma=\sigma_{0}=\frac{N+2 \alpha}{p}$, then $\sigma_{0} \in(0, N)$ if $p>1+\frac{2 \alpha}{N}$. Therefore, there exist some $k>1$ dependent of $\left|c\left(\sigma_{0}, \alpha\right)\right|$ and $c_{N, \alpha}$ but independent of $n$ such that

$$
U(x)=k \Phi_{\sigma_{0}}(x)
$$

is a super solution of (3.2). Thus, $U \in L^{1}\left(B_{1}\left(e_{N}\right)\right)$ and by Theorem 2.1, we have that

$$
0 \leq u_{s} \leq U \text { for any } s \in(0,1) .
$$

For any $x \in \mathbb{R}^{N} \backslash\{0\}, u_{0}(x):=\lim _{s \rightarrow 0^{+}} u_{s}(x) \leq U(x)<+\infty$. Following the same argument of Proposition 2.1, we can prove that $u_{0}$ is a classical solution of (3.2). Furthermore, $u_{0}$ is the minimum solution of (3.2).

Proof of (3.3). Let $\bar{x} \in \partial B_{1}\left(e_{N}\right) \backslash\{0\}, K_{1}=\partial B_{1}\left(e_{N}\right) \cap \overline{B_{|\bar{x}| / 8}(\bar{x})}$ and $K_{2}=\partial B_{1}\left(e_{N}\right) \cap B_{|\bar{x}| / 2}^{c}(\bar{x})$. Let $\mathcal{O}$ be an open and $C^{2}$ set such that

$$
B_{1}\left(e_{N}\right) \cap B_{|\bar{x}| / 4}(\bar{x}) \subset \mathcal{O} \subset B_{1}\left(e_{N}\right) \cap B_{|\bar{x}| / 2}(\bar{x}) .
$$

Then we see that

$$
K_{1} \subset \partial \mathcal{O} \text { and } \partial \mathcal{O} \cap K_{2}=\emptyset .
$$

We would like to find a super solution of (3.2) in $\mathcal{O}$ with vanishing boundary value in $K_{1}$ for any $n$. Denote

$$
V_{\lambda}=U \eta+\lambda V_{\mathcal{O}}
$$

where $U$ is defined (3.7), $\eta$ is a $C^{2}$ function such that

$$
\eta(x)=\left\{\begin{array}{lll}
1 & \text { if } & x \in B_{|\bar{x}| / 4}^{c}(\bar{x}), \\
0 & \text { if } & x \in B_{|\bar{x}| / 8}(\bar{x}) .
\end{array}\right.
$$

and $V_{\mathcal{O}}$ is the solution of

$$
\begin{aligned}
(-\Delta)^{\alpha} u=1 \quad \text { in } \quad \mathcal{O}, \\
u=0 \quad \text { in } \quad \mathcal{O}^{c} .
\end{aligned}
$$

Since $U(1-\eta)$ is $C^{2}$ in $\mathbb{R}^{N}$, then there exists $c_{13}>0$ such that

$$
\left|(-\Delta)^{\alpha} U(1-\eta)\right| \leq c_{13} \quad \text { in } \quad \overline{\mathcal{O}}
$$

Thus, there exists $c_{14}>0$ such that $\left|(-\Delta)^{\alpha} U \eta\right| \leq c_{14}$ in $\overline{\mathcal{O}}$. Choosing $\lambda_{0}>0$ suitable, we have that for $\lambda \geq \lambda_{0}$,

$$
(-\Delta)^{\alpha} V_{\lambda}+V_{\lambda}^{p} \geq \frac{c_{N, \alpha}}{|x|^{N+2 \alpha}} \text { in } \mathcal{O} .
$$

Moreover, since $\eta=1$ in $\mathbb{R}^{N} \backslash \mathcal{O}$, then $V_{\lambda} \geq U \geq u_{s}$ in $\mathbb{R}^{N} \backslash \mathcal{O}$. By the Comparison Principle, we have that for any $s \in(0,1)$,

$$
u_{s} \leq V_{\lambda} \quad \text { in } \quad B_{1}\left(e_{N}\right) .
$$

which implies (3.3).

Proof of (3.4). For $t \in(0,1)$, denote that

$$
V_{t}(x)=c_{N, \alpha}^{\frac{1}{p}} t^{-\frac{N+2 \alpha}{p}} V_{B}\left(\frac{x-t e_{N}}{t}\right), \quad x \in \mathbb{R}^{N},
$$

where $V_{B}$ is the solution of

$$
\begin{array}{rll}
(-\Delta)^{\alpha} u=1 & \text { in } \quad & B_{1}(0), \\
u=0 & \text { in } & \mathbb{R}^{N} \backslash B_{1}(0) .
\end{array}
$$


It deduces by (3.1) that $x \in B_{\frac{t}{4}}\left(t e_{N}\right)$,

$$
(-\Delta)^{\alpha} V_{t}(x)=c_{N, \alpha}^{\frac{1}{p}} t^{-\frac{N+2 \alpha}{p}-2 \alpha} \leq c_{N, \alpha}^{\frac{1}{p}} t^{-N-2 \alpha}
$$

and

$$
\frac{1}{\left|x+t e_{N}\right|^{N+2 \alpha}} \geq \frac{c_{15}}{t^{N+2 \alpha}}
$$

where $c_{15}>0$ independent of $t$. Then there exists some constant $\nu \in(0,1)$ such that

$$
(-\Delta)^{\alpha}\left(\nu V_{t}\right)+\left(\nu V_{t}\right)^{p} \leq \frac{c_{N, \alpha}}{t^{N+2 \alpha}} \leq(-\Delta)^{\alpha} u_{0}+u_{0}^{p} \quad \text { in } \quad B_{\frac{t}{4}}\left(t e_{N}\right)
$$

and $\nu V_{t}=0 \leq u_{0}$ in $B_{\frac{t}{4}}\left(t e_{N}\right)^{c}$. By applying the Comparison Principle, we have that

$$
\nu V_{t} \leq u_{0} \quad \text { in } \quad \mathbb{R}^{N}
$$

which implies

$$
u_{0}(x) \geq \nu c_{N, \alpha}^{\frac{1}{p}} t^{-\frac{N+2 \alpha}{p}} \min _{B_{\frac{1}{2}}(0)} V_{B} \geq c_{16} c_{N, \alpha}^{\frac{1}{p}}|x|^{-\frac{N+2 \alpha}{p}}, \quad \forall x \in B_{\frac{t}{8}}\left(t e_{N}\right),
$$

for some constants $c_{16}>0$ independent of $t$. We complete the proof.

Now we are in the position to prove Theorem 1.1

Proof of Theorem 1.1. By Proposition 3.1, there exists minimum solution $u_{0}$ of (3.2). Using the maximum principle argument, we have that for $s \in(0,1)$,

$$
u_{s} \leq u_{0} \quad \text { in } \quad B_{1}\left(e_{N}\right)
$$

Since

$$
u_{0} \in L^{1}\left(B_{1}\left(e_{N}\right)\right), \quad u_{0}^{p} \in L^{1}\left(B_{1}\left(e_{N}\right), \rho^{2} d x\right),
$$

where $\rho(x)=\operatorname{dist}\left(x, \partial B_{1}\left(e_{N}\right)\right)$, then there exists $\tilde{u}_{0} \leq u_{0}$ such that

$$
u_{s} \rightarrow \tilde{u}_{0} \quad \text { in } B_{1}\left(e_{N}\right) \text { and in } L^{1}\left(B_{1}\left(e_{N}\right)\right)
$$

and

$$
u_{s}^{p} \rightarrow \tilde{u}_{0}^{p} \quad \text { in } B_{1}\left(e_{N}\right) \text { and in } L^{1}\left(B_{1}\left(e_{N}\right), \rho^{2} d x\right) .
$$

Passing the limit in identity of (2.13) as $s \rightarrow 0^{+}$, we have that

$$
\int_{B_{1}\left(e_{N}\right)}\left[\tilde{u}_{0}(-\Delta)^{\alpha} \xi+\tilde{u}_{0}^{p} \xi\right] d x=\int_{B_{1}\left(e_{N}\right)} \xi \Gamma_{0} d x, \quad \forall \xi \in C_{0}^{\infty}\left(B_{1}\left(e_{N}\right)\right) .
$$

By the same argument, we have that $\tilde{u}_{0}$ is $C^{2}$ locally in $B_{1}\left(e_{N}\right)$ and $\tilde{u}_{0} \chi_{B_{1}\left(e_{N}\right)}$ is the minimum solution of (3.2). Thus, we have

$$
\tilde{u}_{0}=u_{0} \quad \text { in } \quad B_{1}\left(e_{N}\right) .
$$

Uniqueness. Let $v_{0}$ be a weak solution of (2.2) and then $\varphi_{0}:=u_{0}-v_{0}$ is a weak solution of

$$
\begin{array}{rll}
(-\Delta)^{\alpha} \varphi_{0}+u_{0}^{p}-v_{0}^{p}=0 & \text { in } \quad B_{1}\left(e_{N}\right) \\
\varphi_{0}=0 & \text { in } \quad & \mathbb{R}^{N} \backslash B_{1}\left(e_{N}\right),
\end{array}
$$

that is,

$$
\int_{B_{1}\left(e_{N}\right)} \varphi_{0}(-\Delta)^{\alpha} \xi d x+\int_{B_{1}\left(e_{N}\right)}\left(u_{0}^{p}-v_{0}^{p}\right) \xi d x=0, \quad \forall \xi \in C_{0}^{\infty}\left(B_{1}\left(e_{N}\right)\right) .
$$


In this definition of weak solution, we can not apply Kato's inequality [9, Proposition 2.4] directly due to the stronger test functions space. So we have to improve the regularity of $\varphi_{0}$. In fact, for any $\xi \in \mathbb{X}_{\alpha, B_{1}\left(e_{N}\right)}$, let $\left\{\xi_{k}\right\}$ be a sequence of nonnegative functions in $C_{0}^{\infty}\left(B_{1}\left(e_{N}\right)\right)$, such that

$$
\xi_{k} \rightarrow \xi \quad \text { and } \quad\left|(-\Delta)^{\alpha} \xi_{k}\right| \leq 2\left|(-\Delta)^{\alpha} \xi\right| \quad \text { in } \quad B_{1}\left(e_{N}\right) .
$$

Passing the limit of (3.13) with $\xi_{k}$ as $k \rightarrow \infty$, we have that (3.13) holds for any $\xi \in \mathbb{X}_{\alpha, B_{1}\left(e_{N}\right)}$. If we choose a sequence $\left\{\tilde{\xi}_{k}\right\} \subset \mathbb{X}_{\alpha, B_{1}\left(e_{N}\right)}$ which converges to $\operatorname{sign}\left(u_{0}^{p}-v_{0}^{p}\right) \cdot \mathbb{G}_{\alpha, B_{1}\left(e_{N}\right)}[1]$ and then there exists $c_{6}>0$ such that

$$
\int_{B_{1}\left(e_{N}\right)}\left|u_{0}^{p}-v_{0}^{p}\right| \mathbb{G}_{\alpha, B_{1}\left(e_{N}\right)}[1] d x \leq c_{6}\left\|\varphi_{0}\right\|_{L^{1}\left(B_{1}\left(e_{N}\right)\right)},
$$

therefore, $\left|u_{0}^{p}-v_{0}^{p}\right| \in L^{1}\left(B_{1}\left(e_{N}\right), \rho^{\alpha} d x\right)$.

Now we follows the Kato's inequality [9, Proposition 2.4] to obtain that

$$
\int_{B_{1}\left(e_{N}\right)}\left|\varphi_{0}\right|(-\Delta)^{\alpha} \xi+\int_{B_{1}\left(e_{N}\right)}\left[u_{0}^{p}-v_{0}^{p}\right] \operatorname{sign}\left(u_{0}-v_{0}\right) \xi d x=0, \quad \forall \xi \in \mathbb{X}_{\alpha, B_{1}\left(e_{N}\right)} .
$$

Taking $\xi=\mathbb{G}_{\alpha}[1]$, we have that

$$
\int_{B_{1}\left(e_{N}\right)}\left[u_{0}^{p}-v_{0}^{p}\right] \operatorname{sign}\left(u_{0}-v_{0}\right) \xi d x \geq 0 \quad \text { and } \quad \int_{B_{1}\left(e_{N}\right)}\left|\varphi_{0}\right| d x=0,
$$

then $\varphi_{0}=0$ a.e. in $B_{1}\left(e_{N}\right)$. Then the uniqueness is proved.

\section{Nonexistence}

In order to prove the nonexistence of weak solution to (1.1) for $p \in\left(0,1+\frac{2 \alpha}{N}\right]$, we will prove that the solution $u_{s}$ of problem (2.1) blows up in $B_{1}\left(e_{N}\right)$ as $s \rightarrow 0^{+}$. To this end, we first define a cone by

$$
\mathcal{C}=\left\{x \in \mathbb{R}^{N}: \exists t \in(0,1) \text { s.t. }\left|x-t e_{N}\right|<\frac{t}{8}\right\} .
$$

We observe that $\mathcal{C} \subset B_{1}\left(e_{N}\right)$.

Lemma 4.1 Assume that $p>0, s \in(0,1)$ and $u_{s}$ is the unique solution of problem (2.1). Then there exists $c_{17}>0$ such that

$$
\lim _{s \rightarrow 0^{+}} u_{s}(x) \geq c_{17}|x|^{-N}, \quad \forall x \in \mathcal{C} .
$$

Proof. By Proposition 2.1, we have that $\tilde{u}_{s}:=u_{s} \chi_{B_{1}\left(e_{N}\right)}$ is the unique classical solution of (2.8)). For given $t \in(0,1)$, we recall

$$
V_{t}(x)=t^{-N} V_{B}\left(\frac{x-t e_{N}}{t}\right),
$$

where $V_{B}$ is the solution of (3.10).

We see that for $x \in B_{\frac{t}{4}}\left(t e_{N}\right)$,

$$
(-\Delta)^{\alpha} V_{t}(x)=t^{-N-2 \alpha}
$$

and choose $s \leq \frac{t}{4}$, then

$$
\left|x+s e_{N}\right| \leq|x|+s \leq \frac{3}{2} t
$$

which implies that

$$
\frac{1}{\left|x+s e_{N}\right|^{N+2 \alpha}} \geq \frac{c_{18}}{t^{N+2 \alpha}}
$$


where $c_{18}>0$ independent of $t$. Then there exists some constant $\nu>0$ such that

$$
(-\Delta)^{\alpha}\left(\nu V_{t}\right)+\left(\nu V_{t}\right)^{p} \leq \frac{c_{N, \alpha}}{t^{N+2 \alpha}} \leq(-\Delta)^{\alpha} \tilde{u}_{s}+\tilde{u}_{s}^{p} \quad \text { in } \quad B_{\frac{t}{4}}\left(t e_{N}\right)
$$

and $\nu V_{t}=0 \leq \tilde{u}_{s}$ in $B_{\frac{t}{4}}\left(t e_{N}\right)^{c}$, by applying the Comparison Principle, we have that

$$
V_{t} \leq \tilde{u}_{s} \quad \text { in } \quad \mathbb{R}^{N}
$$

which implies

$$
u_{s}(x) \geq \nu t^{-N} \min _{B_{\frac{1}{2}}(0)} V_{B} \geq c_{19}|x|^{-N}, \quad \forall x \in B_{\frac{t}{8}}\left(t e_{N}\right),
$$

for some constants $c_{19}>0$ independent of $t$ and $s$. Combining the increasing monotonicity, we have that

$$
\lim _{s \rightarrow 0^{+}} u_{s}(x) \geq c_{19}|x|^{-N}, \quad \forall x \in \mathcal{C} .
$$

Lemma 4.2 Assume that $p \geq 0, s \in(0,1)$ and $\tilde{u}_{s}:=u_{s} \chi_{B_{1}\left(e_{N}\right)}$ is the minimal solution of (2.8). Then

(i) $\tilde{u}_{s}\left(x^{\prime}, x_{N}\right)$ is symmetric with respect to $x^{\prime}$ and decreasing with to $r^{\prime}:=\left|x^{\prime}\right|$ for any $x_{N} \in(0,2)$;

(ii) $\tilde{u}_{s}\left(x^{\prime}, x_{N}\right)$ is decreasing in $x_{N}$ for $x_{N} \in\left(1,1+\sqrt{1-\left|x^{\prime}\right|^{2}}\right)$.

Proof. By applying the same procedure of step 1 and step 2 in proof of Theorem 1.1 in [14], we have that

$$
u_{s}\left(x^{\prime}, x_{N}\right)=u_{s}\left(\left|x^{\prime}\right|, x_{N}\right) \quad \text { for } \quad x=\left(x^{\prime}, x_{N}\right) \in B_{1}\left(e_{N}\right)
$$

and $u_{s}\left(r, x_{N}\right)$ is decreasing with $r=\left|x^{\prime}\right|$. Using the same argument from the other side, we conclude that $u_{s}\left(x^{\prime}, x_{N}\right) \leq u_{s}\left(x^{\prime}, 2-x_{N}\right)$ for $x_{N} \in(1,2)$, that is, $u_{s}\left(x^{\prime}, x_{N}\right)$ is decreasing with $x_{N} \in(1,2)$.

Proof of Theorem 1.2. For $p=0$, the solution $u_{s}$ of (2.1) satisfies

$$
u_{s}=\mathbb{G}_{\alpha}\left[\Gamma_{s}\right]-\mathbb{G}_{\alpha}[1] .
$$

By Lemma 3.2 , we know that $\mathbb{G}_{\alpha}[\cdot]$ blows every where in $B_{1}\left(e_{N}\right)$, but $\mathbb{G}_{\alpha}[1]$ is bounded uniformly. For $0<p \leq 1+\frac{2 \alpha}{N}$, our proof is divided into two steps.

Step 1. we prove that

$$
\lim _{s \rightarrow 0^{+}} u_{s}(x)=+\infty, \quad x \in \mathcal{B}:=\left\{\left(x^{\prime}, x_{N}\right) \in B_{1}\left(e_{N}\right): x_{N}>1\right\} .
$$

If there is $\bar{x}=\left(\bar{x}_{0}, t_{0}\right) \in \mathcal{B}$ such that $\lim _{s \rightarrow 0^{+}} u_{s}(\bar{x})<+\infty$, then by Lemma 4.2, we have that

$$
\lim _{s \rightarrow 0^{+}} u_{s}(x) \leq \lim _{s \rightarrow 0^{+}} u_{s}(\bar{x}) \quad \text { in } \quad B_{+}:=\left\{x=\left(x^{\prime}, x_{N}\right) \in B_{1}\left(e_{N}\right):\left|x^{\prime}\right|>\left|\bar{x}^{\prime}\right|, x_{N}>t_{0}\right\} .
$$

Choose a nonnegative function $\xi_{0} \in C_{0}^{\infty}$ with support in $B_{+}$, then for any $x \in B_{1}\left(e_{N}\right) \backslash B_{+}$,

$$
\begin{aligned}
(-\Delta)^{\alpha} \xi_{0}(x) & =-c_{N, \alpha} \mathrm{P} \cdot \mathrm{V} \cdot \int_{\mathbb{R}^{N}} \frac{\xi_{0}(y)-\xi_{0}(x)}{|x-y|^{N+2 \alpha}} d y \\
& =-c_{N, \alpha} \int_{B_{+}} \frac{\xi_{0}(y)}{|x-y|^{N+2 \alpha}} d y \\
& \leq-c_{N, \alpha}\left|x-2 e_{N}\right|^{-N-2 \alpha} \int_{B_{+}} \xi_{0}(y) d y
\end{aligned}
$$

therefore, there exists $c_{20}>0$ such that

$$
(-\Delta)^{\alpha} \xi_{0}(x) \leq-c_{20}, \quad \forall x \in B_{1}\left(e_{N}\right) \backslash B_{+} .
$$


Moreover, there exists $c_{21}>0$ such that

$$
\left|(-\Delta)^{\alpha} \xi_{0}(x)\right| \leq c_{21}, \quad \forall x \in B_{+} .
$$

Since $\mathcal{C} \subset\left(B_{1}\left(e_{N}\right) \backslash B_{+}\right)$, then by Lemma 4.1 we have that

$$
\begin{aligned}
\int_{B_{1}\left(e_{N}\right)} u_{s}(-\Delta)^{\alpha} \xi_{0} d x \leq & \int_{\mathcal{C}} u_{s}(-\Delta)^{\alpha} \xi_{0} d x+\int_{B_{1}\left(e_{N}\right) \backslash\left(B_{+} \cup \mathcal{C}\right)} u_{s}(-\Delta)^{\alpha} \xi_{0} d x \\
& +\int_{B_{+}} u_{s}\left|(-\Delta)^{\alpha} \xi_{0}\right| d x \\
\leq & -c_{20} \int_{\mathcal{C}} u_{s} d x+c_{21}\left|B_{+}\right| \\
\rightarrow & -\infty \text { as } s \rightarrow 0^{+}
\end{aligned}
$$

While

$$
\left|\int_{B_{1}\left(e_{N}\right)} u_{s}^{p} \xi_{0} d x\right|=\int_{B_{+}} u_{s}^{p} \xi_{0} d x \leq u_{s}^{p}(\bar{x}) \max _{\mathbb{R}^{N}} \xi_{0}\left|B_{+}\right|
$$

and

$$
\int_{B_{1}\left(e_{N}\right)} \frac{\xi_{0}(x)}{\left|x+s e_{N}\right|^{N+2 \alpha}} d x=\int_{B_{+}} \frac{\xi_{0}(x)}{\left|x+s e_{N}\right|^{N+2 \alpha}} d x \leq\left|B_{+}\right| \max _{\mathbb{R}^{N}} \xi_{0}
$$

where $\left|x+s e_{N}\right| \geq 1$ for $x \in B_{+}$. Then taking $s>0$ small enough, we obtain a contradiction with the identity

$$
\int_{B_{1}\left(e_{N}\right)}\left[u_{s}(-\Delta)^{\alpha} \xi+u_{s}^{p} \xi\right] d x=c_{N, \alpha} \int_{B_{1}\left(e_{N}\right)} \frac{\xi(x)}{\left|x+s e_{N}\right|^{N+2 \alpha}} d x, \quad \forall \xi \in C_{0}^{\infty}\left(B_{1}\left(e_{N}\right)\right) .
$$

Therefore,

$$
\lim _{s \rightarrow 0^{+}} u_{s}(x)=+\infty, \quad \forall x \in \mathcal{B} .
$$

Step 2: We claim that $\lim _{s \rightarrow 0^{+}} u_{s}(x)=\infty, x \in B_{1}\left(e_{N}\right)$. By the fact of $\lim _{s \rightarrow 0^{+}} u_{s}(x)=\infty$, $x \in \mathcal{B}$, then letting $\tilde{x}=\left(\frac{1}{2}, 0 \cdots, \frac{3}{2}\right)$, for any $n>1$, there exists $s_{n}>0$ such that $s_{n} \rightarrow 0$ as $n \rightarrow+\infty$ and

$$
u_{s_{n}}(\tilde{x}) \geq n
$$

then applying Lemma 4.2, we have that

$$
u_{s_{n}} \geq n \quad \text { in } \quad \mathcal{B}_{0}=\left\{x=\left(x^{\prime}, x_{N}\right) \in \mathcal{B}:\left|x^{\prime}\right| \leq \frac{1}{2}, 1 \leq x_{N} \leq \frac{3}{2}\right\} .
$$

For any $x_{0} \in B_{1}\left(e_{N}\right) \backslash \mathcal{B}$, there exists $r_{1}>0$ such that $\bar{B}_{r_{1}}\left(x_{0}\right) \subset B_{1}\left(e_{N}\right) \backslash \mathcal{B}$. We denote by $\phi_{n}$ the solution of

$$
\begin{array}{rll}
(-\Delta)^{\alpha} u+u^{p}=0 & \text { in } & B_{r_{1}}\left(x_{0}\right), \\
u=0 & \text { in } & B_{r_{1}}^{c}\left(x_{0}\right) \backslash \mathcal{B}_{0}, \\
u=n & \text { in } & \mathcal{B}_{0} .
\end{array}
$$

Then by Theorem 2.1, we have that

$$
u_{s_{n}} \geq \phi_{n} \quad \text { in } \quad B_{1}\left(e_{N}\right) .
$$

Let $\varphi_{n}=\phi_{n}-n \chi_{\mathcal{B}_{0}}$, then $\varphi_{n}=\phi_{n}$ in $B_{r_{1}}\left(x_{0}\right)$ and

$$
\begin{aligned}
(-\Delta)^{\alpha} \varphi_{n}(x)+\varphi_{n}^{p}(x) & =(-\Delta)^{\alpha} \phi_{n}(x)-n(-\Delta)^{\alpha} \chi_{\mathcal{B}_{0}}(x)+\phi_{n}^{p}(x) \\
& =n \int_{\mathcal{B}_{0}} \frac{d y}{|y-x|^{N+2 \alpha}}, \quad \forall x \in B_{r_{1}}\left(x_{0}\right),
\end{aligned}
$$


that is, $\varphi_{n}$ is a solution of

$$
\begin{aligned}
(-\Delta)^{\alpha} u+u^{p} & =n \int_{\mathcal{B}_{0}} \frac{d y}{|y-x|^{N+2 \alpha}} & \text { in } & B_{r_{1}}\left(x_{0}\right), \\
u & =0 & \text { in } & B_{r_{1}}^{c}\left(x_{0}\right) .
\end{aligned}
$$

By direct computation,

$$
\frac{1}{c_{23}} \leq \int_{\mathcal{B}_{0}} \frac{d y}{|y-x|^{N+2 \alpha}} \leq c_{23}, \quad \forall x \in B_{r_{1}}\left(x_{0}\right)
$$

for some $c_{23}>1$.

Let $\eta_{1}$ be the solution of

$$
\begin{array}{rll}
(-\Delta)^{\alpha} u=1 & \text { in } & B_{r_{1}}\left(x_{0}\right), \\
u=0 & \text { in } \quad B_{r_{1}}^{c}\left(x_{0}\right)
\end{array}
$$

and then $\left(\frac{n}{2 c_{23}}\right)^{\frac{1}{p}} \max \eta_{1} \cdot \eta_{1}$ is sub solution of (4.5) for $n$ large enough. Then it infers by Theorem 2.1 that

$$
\varphi_{n} \geq\left(\frac{n}{2 c_{23}}\right)^{\frac{1}{p}} \max \eta_{1} \cdot \eta_{1}, \quad \forall x \in B_{r_{1}}\left(x_{0}\right),
$$

which implies that

$$
\phi_{n} \geq\left(\frac{n}{2 c_{23}}\right)^{\frac{1}{p}} \max \eta_{1} \cdot \eta_{1}, \quad \forall x \in B_{r_{1}}\left(x_{0}\right) .
$$

Then by (4.4),

$$
\lim _{s \rightarrow 0^{+}} u_{s_{n}}\left(x_{0}\right) \geq \lim _{n \rightarrow \infty} \phi_{n}\left(x_{0}\right)=\infty .
$$

Since $x_{0}$ is arbitrary in $B_{1}\left(e_{N}\right) \backslash \mathcal{B}$, it implies that $\lim _{n \rightarrow \infty^{+}} u_{s_{n}}(x)=\infty$ in $B_{1}\left(e_{N}\right)$.

\section{The solutions vanishes as $\alpha \rightarrow 1^{-}$}

\subsection{The case of $p \geq \frac{N+2}{N-2}$}

Lemma 5.1 (i) Let $N \geq 2$ and $c_{N, \alpha}$ define in (1.2). Then

$$
\lim _{\alpha \rightarrow 1^{-}} \frac{c_{N, \alpha}}{1-\alpha}=\frac{4 N}{\left|S^{N-1}\right|}
$$

where $\left|S^{N-1}\right|$ denotes the $(N-1)$-dimensional measure of the unit sphere $S^{N-1}$.

(ii) Let $N \geq 2$, then for any $f \in C_{0}^{\infty}\left(\mathbb{R}^{N}\right)$,

$$
\lim _{\alpha \rightarrow 1^{-}}(-\Delta)^{\alpha} f=-\Delta f
$$

Proof. The proofs of $(i)$ and $(i i)$ see Corollary 4.2 and Proposition 4.4 in [27] respectively.

Proposition 5.1 Let $\Phi_{\sigma}$ be defined by (3.5) with $\sigma \in(0, N)$ and

$$
c(\sigma, \alpha)=-\frac{1}{2} \int_{\mathbb{R}^{N}} \frac{\left|z+e_{N}\right|^{-\sigma}+\left|z-e_{N}\right|^{-\sigma}-2}{|z|^{N+2 \alpha}} d z .
$$

Then

$$
(-\Delta)^{\alpha} \Phi_{\sigma}(x)=\frac{c(\sigma, \alpha)}{|x|^{\sigma+2 \alpha}}, \quad \forall x \in \mathbb{R}^{N} \backslash\{0\}
$$

Moveover,

$$
\lim _{\alpha \rightarrow 1^{-}} c(\sigma, \alpha)=(N-2-\sigma) \sigma
$$

and

$$
\lim _{\alpha \rightarrow 1^{-}}(-\Delta)^{\alpha} \Phi_{\sigma}(x)=\frac{(N-2-\sigma) \sigma}{|x|^{\sigma+2}}, \quad \forall x \in \mathbb{R}^{N} \backslash\{0\} .
$$


Proof. By direct computation, we have that

$$
(-\Delta)^{\alpha} \Phi_{\sigma}(x)=\frac{c(\sigma, \alpha)}{|x|^{\sigma+2 \alpha}}, \quad \forall x \in \mathbb{R}^{N} \backslash\{0\}
$$

where

$$
c(\sigma, \alpha)=-\frac{1}{2} \int_{\mathbb{R}^{N}} \frac{\frac{1}{\left|z+e_{N}\right|^{\sigma}}+\frac{1}{\left|z-e_{N}\right|^{\sigma}}-2}{|z|^{N+2 \alpha}} d z .
$$

On the other hand, it has been proved in [4] that

$$
c(\sigma, \alpha)=0 \quad \text { if } \quad \sigma=N-2 \alpha .
$$

Now for any $R>1$, let $\eta_{R}: \mathbb{R}^{N} \rightarrow[0,1]$ be a nonnegative function such that $\eta_{R}=1$ in $B_{R}(0) \backslash B_{\frac{1}{R}}(0)$ and $\eta_{R}=0$ in $B_{2 R}^{c}(0) \cup B_{\frac{1}{2 R}}(0)$, then $\eta_{R} \Phi_{\sigma} \in C_{0}^{\infty}\left(\mathbb{R}^{N}\right)$. By Lemma 5.1 (ii), we have

$$
\lim _{\alpha \rightarrow 1^{-}}(-\Delta)^{\alpha}\left(\eta_{R} \Phi_{\sigma}\right)\left(e_{N}\right)=(N-2-\sigma) \sigma
$$

and

$$
(-\Delta)^{\alpha}\left(\eta_{R} \Phi_{\sigma}\right)\left(e_{N}\right)=(-\Delta)^{\alpha} \Phi_{\sigma}\left(e_{N}\right)-c_{N, \alpha} \int_{\mathbb{R}^{N}} \frac{\left(1-\eta_{R}(z)\right)|z|^{-\sigma}}{\left|z-e_{N}\right|^{N+2 \alpha}} d z .
$$

A straightforward computation implies that

$$
\begin{aligned}
0 & \leq \int_{\mathbb{R}^{N}} \frac{\left(1-\eta_{R}(z)\right)|z|^{-\sigma}}{\left|z-e_{N}\right|^{N+2 \alpha}} d z \\
& \leq \int_{B_{\frac{1}{R}}(0) \cup B_{R}^{c}(0)} \frac{|z|^{-\sigma}}{\left|z-e_{N}\right|^{N+2 \alpha}} d z \\
& \leq c_{24} \int_{B_{\frac{1}{R}}(0) \cup B_{R}^{c}(0)} \frac{|z|^{-\sigma}}{\left|z-e_{N}\right|^{N}} d z,
\end{aligned}
$$

where $c_{24}>0$ is independent of $\alpha$. Thus,

$$
\lim _{\alpha \rightarrow 1^{-}} c_{N, \alpha} \int_{\mathbb{R}^{N}} \frac{\left(1-\eta_{R}(z)\right)|z|^{-\sigma}}{\left|z-e_{N}\right|^{N+2 \alpha}} d z=0
$$

Therefore, passing to the limit of (5.4) as $\alpha \rightarrow 1^{-}$, we conclude that $\lim _{\alpha \rightarrow 1^{-}} c(\sigma, \alpha)=(N-2-\sigma) \sigma$ and combining (5.3), (5.2) holds.

Proposition 5.2 Let $p \geq \frac{N+2}{N-2}, \sigma_{p}=\frac{N+2}{p}$ and $u_{\alpha, p}$ be the unique weak solution of (1.1). Then

$$
u_{\alpha, p} \leq\left(4^{1-\alpha} c_{N, \alpha}\right)^{\frac{1}{p}} \Phi_{\sigma_{p}} \quad \text { in } B_{1}\left(e_{N}\right)
$$

Proof. It follows by (5.1) that

$$
(-\Delta)^{\alpha} \Phi_{\sigma_{p}}(x)=\frac{c\left(\sigma_{p}, \alpha\right)}{|x|^{\sigma_{p}+2 \alpha}}, \quad \forall x \in \mathbb{R}^{N} \backslash\{0\},
$$

where $c\left(\sigma_{p}, \alpha\right) \rightarrow N-2-\sigma_{p}$ as $\alpha \rightarrow 1^{-}$. For $k>1$, denote

$$
U_{k}=k \Phi_{\sigma_{p}} .
$$

When $p>\frac{N+2}{N-2}$, we have $N-2-\sigma_{p}>0$, then there exists $\alpha_{p} \in(0,1)$ such that $c\left(\sigma_{p}, \alpha\right) \geq 0$ for $\alpha \in\left(\alpha_{p}, 1\right)$. When $p=\frac{N+2}{N-2}$, we have $\sigma_{p}=N-2<N-2 \alpha$. Using [13], $c(\cdot, \alpha)$ is $C^{2}$ and convex in $[0, N)$ and

$$
\lim _{\sigma \rightarrow N^{-}} c(\sigma, \alpha)=-\infty
$$


and for $\sigma \in(0, N-2 \alpha)$,

$$
c(\sigma, \alpha) \geq c(N-2 \alpha, \alpha)=0 .
$$

Thus, $c\left(\sigma_{p}, \alpha\right) \geq 0$ by the fact of $\sigma_{p}<N-2 \alpha$. Choosing $k=\left(4^{1-\alpha} c_{N, \alpha}\right)^{\frac{1}{p}}$, then for any $x \in B_{1}\left(e_{N}\right)$,

$$
\begin{aligned}
(-\Delta)^{\alpha} U_{k}+U_{k}^{p}-\frac{c_{N, \alpha}}{|x|^{N+2 \alpha}} & =\frac{k c\left(\sigma_{p}, \alpha\right)}{|x|^{\sigma_{p}+2 \alpha}}+\frac{k^{p}}{|x|^{N+2}}-\frac{c_{N, \alpha}}{|x|^{N+2 \alpha}} \\
& \geq\left(k^{p}-4^{1-\alpha} c_{N, \alpha}\right) \frac{1}{|x|^{N+2}}=0,
\end{aligned}
$$

where we used that $\frac{1}{|x|^{N+2 \alpha}} \leq \frac{4^{1-\alpha}}{|x|^{N+2}}$ for $x \in B_{1}\left(e_{N}\right)$. Therefore, for $\alpha \in\left(\alpha_{p}, 1\right)$ we have that

$$
u_{\alpha, p}(x) \leq\left(4^{1-\alpha} c_{N, \alpha}\right)^{\frac{1}{p}} \Phi_{\sigma_{p}}(x), \quad \forall x \in B_{1}\left(e_{N}\right) .
$$

Proof of Theorem 1.3. It follows by Proposition 5.2 that

$$
u_{\alpha, p} \leq\left(4^{1-\alpha} c_{N, \alpha}\right)^{\frac{1}{p}} \Phi_{\sigma_{p}} \text { in } B_{1}\left(e_{N}\right) .
$$

On the other hand, we deduce from Lemma 5.1 that

$$
c_{N, \alpha} \leq c_{25}(1-\alpha),
$$

where $c_{25}>0$ is independent of $\alpha$. Therefore, we have that

$$
0 \leq \lim _{\alpha \rightarrow 1^{-}} u_{\alpha, p}(x) \leq c_{26} \lim _{\alpha \rightarrow 1^{-}}(1-\alpha)^{\frac{1}{p}} \Phi_{\sigma_{p}}(x)=0, \quad \forall x \in B_{1}\left(e_{N}\right),
$$

where $c_{26}>0$ is independent of $\alpha$. This ends the proof.

\subsection{The case $p \in\left[\frac{N+1}{N-1}, \frac{N+2}{N-2}\right)$}

We know that the unique weak solution $u_{\alpha, p}$ of (1.1) satisfies the identity

$$
\int_{B_{1}\left(e_{N}\right)}\left[u_{\alpha, p}(x)(-\Delta)^{\alpha} \xi(x)+u_{\alpha, p}^{p}(x) \xi(x)\right] d x=\int_{B_{1}\left(e_{N}\right)} \xi(x) \Gamma_{0, \alpha}(x) d x, \quad \forall \xi \in C_{0}^{\infty}\left(B_{1}\left(e_{N}\right)\right) .
$$

From Lemma 5.1 part (ii), it infers that for any $\xi \in C_{0}^{\infty}\left(B_{1}\left(e_{N}\right)\right)$,

$$
(-\Delta)^{\alpha} \xi \rightarrow-\Delta \xi \quad \text { uniformly in } \quad B_{1}\left(e_{N}\right) \text { as } \alpha \rightarrow 1^{-} .
$$

Lemma 5.2 Let $\frac{N+1}{N-1} \leq p<\frac{N+2}{N-2}, \sigma_{p}=\frac{N+2}{p}$ and $u_{\alpha, p}$ be the unique weak solution of (1.1). Then there exists $c_{27}>0$ independent of $\alpha$ such that

$$
0 \leq u_{\alpha, p} \leq c_{27} \Phi_{\sigma_{p}} \quad \text { in } B_{1}\left(e_{N}\right)
$$

Proof. For $\frac{N+1}{N-1} \leq p<\frac{N+2}{N-2}$, we have $\sigma_{p}<N$. It follows by (5.1) that

$$
(-\Delta)^{\alpha} \Phi_{\sigma_{p}}(x)=\frac{c\left(\sigma_{p}, \alpha\right)}{|x|^{\sigma_{p}+2 \alpha}}, \quad \forall x \in \mathbb{R}^{N} \backslash\{0\},
$$

where $c\left(\sigma_{p}, \alpha\right) \rightarrow N-2-\sigma_{p}$ as $\alpha \rightarrow 1^{-}$. There exists $\alpha_{p} \in(0,1)$ such that

$$
\left|c\left(\sigma_{p}, \alpha\right)\right| \leq 2\left|N-2-\sigma_{p}\right|, \quad \alpha \in\left(\alpha_{p}, 1\right) .
$$

For $k>1$, denote

$$
U_{k}=k \Phi_{\sigma_{p}}
$$


For $\alpha \in\left(\alpha_{p}, 1\right)$ and any $x \in B_{1}\left(e_{N}\right)$, we deduce that

$$
\begin{aligned}
(-\Delta)^{\alpha} U_{k}+U_{k}^{p}-\frac{c_{N, \alpha}}{|x|^{N+2 \alpha}} & \geq-\frac{2 k\left|N-2-\sigma_{p}\right|}{|x|^{\sigma_{p}+2 \alpha}}+\frac{k^{p}}{|x|^{N+2}}-\frac{c_{N, \alpha}}{|x|^{N+2 \alpha}} \\
& \geq\left(k^{p}-4^{1-\alpha} c_{N, \alpha}-2^{N+3-\sigma_{p}} k\left|N-2-\sigma_{p}\right|\right) \frac{1}{|x|^{N+2}},
\end{aligned}
$$

which implies that there exists $k_{0}>0$ independent of $\alpha$ such that for $\alpha \in\left(\alpha_{p}, 1\right)$ we have that

$$
u_{\alpha, p}(x) \leq k_{0} \Phi_{\sigma_{p}}(x), \quad \forall x \in B_{1}\left(e_{N}\right) .
$$

We have that $\Phi_{\sigma_{p}} \in L^{1}\left(B_{1}\left(e_{N}\right)\right)$ and

$$
\left\|\Phi_{\sigma_{p}}\right\|_{M^{p^{*}}\left(B_{1}(0), d x\right)} \leq c_{28}
$$

where $c_{28}>0, p^{*}=\frac{N}{\sigma_{p}}>1$ and $M^{p^{*}}\left(B_{1}(0)\right)$ is the Marcinkiewicz space of exponent $p^{*}$. By regularity results, we have that

$$
\left\|u_{\alpha, p}\right\|_{C_{\mathrm{loc}}^{\beta}\left(B_{1}(0)\right)} \leq c_{29}
$$

where $c_{29}>0$ is independent of $\alpha$ by (5.8). Therefore, by compactness, we only have to prove that 0 is the only accumulation point of the sequence $\left\{u_{\alpha, p}\right\}_{\alpha}$. Let $u^{*}$ in $L^{1}\left(B_{1}\left(e_{N}\right)\right)$ be accumulation point of the sequence $\left\{u_{\alpha, p}\right\}_{\alpha}$ and a subsequence, still denote $\left\{u_{\alpha, p}\right\}_{\alpha}$, converge to $u^{*}$.

On the one hand, $\Gamma_{0, \alpha}$ converges to 0 uniformly in any compact subset of $B_{1}\left(e_{N}\right)$. Therefore, for $\xi \in C_{0}^{\infty}\left(B_{1}(0)\right)$, we have that

$$
\lim _{\alpha \rightarrow 1^{-}} \int_{B_{1}\left(e_{N}\right)} \xi(x) \Gamma_{0, \alpha}(x) d x=0 .
$$

Thus, we have that $u^{*}$ satisfies the identity

$$
\int_{B_{1}\left(e_{N}\right)}\left[u^{*}(x)(-\Delta) \xi(x)+\left(u^{*}\right)^{p}(x) \xi(x)\right] d x=0, \quad \forall \xi \in C_{0}^{\infty}\left(B_{1}\left(e_{N}\right)\right),
$$

On the other hand, by regularity result, we have that $u^{*} \in C^{2}\left(B_{1}\left(e_{N}\right)\right) \cap L^{1}\left(B_{1}\left(e_{N}\right)\right)$ continuous up to boundary $\partial B_{1}\left(e_{N}\right) \backslash\{0\}$, therefore, $u^{*}$ is a nonnegative classical solution of

$$
\begin{aligned}
-\Delta u+u^{p}=0 & \text { in } B_{1}\left(e_{N}\right), \\
u=0 & \text { on } \partial B_{1}\left(e_{N}\right) \backslash\{0\} .
\end{aligned}
$$

Then from Theorem 3.1 in [15], we have that

$$
u^{*} \in L^{\infty}\left(B_{1}(0)\right)
$$

and Theorem 3.1 in [15], $u^{*}$ is a classical solution of

$$
\begin{aligned}
-\Delta u+u^{p}=0 & \text { in } \quad B_{1}\left(e_{N}\right), \\
u=0 & \text { on } \quad \partial B_{1}\left(e_{N}\right),
\end{aligned}
$$

where $p \in\left(1, \frac{N+1}{N-1}\right)$. By Strong Maximum Principle, we have that

$$
u^{*} \equiv 0 \quad \text { in } \quad B_{1}\left(e_{N}\right) .
$$

Acknowledgements: The authors want to thank the referee for carefully reading the paper and making suggestions that resulted in a great improvement in the clarity of the proofs. H. Chen is supported by National Natural Science Foundation of China, No:11401270 and the Projectsponsored by SRF for ROCS, SEM. 


\section{References}

[1] D. Balenu, K. Diethelm, E. Scalas and J. Trujillo, Fractional calculus models and numerical methods, Amsterdam, Word Science Publish, Nonlinear Science and complexity.

[2] M. Bidaut-Véron, A. C. Ponce and L. Véron, Isolated boundary singularities of semilinear elliptic equations, arXiv:0902.0449.

[3] M. F. Bidaut-Véron and C. Yarur, Semilinear elliptic equations and systems with measure data: existence and a priori estimates, Advances in Differential Equations 7(3), 257-296 (2002).

[4] L. Caffarelli and L. Silvestre, An extension problem related to the fractional Laplacian, Commun. PDE 32, 1245-1260 (2007).

[5] L. Caffarelli and L. Silvestre, Regularity theory for fully nonlinear integro-differential equations, Comm. Pure Appl. Math. 62, 597-638 (2009).

[6] H. Chen, P. Felmer and A. Quaas, Large solution to elliptic equations involving fractional Laplacian, Ann. Inst. H. Poincaré, Analyse Non Linéaire, doi:10.1016/j.anihpc.2014.08.001.

[7] H. Chen and H. Hajaiaj, Existence, Non-existence, Uniqueness of solutions for semilinear elliptic equations involving measures concentrated on boundary, arXiv:1410.2672.

[8] Z. Chen and R. Song, Estimates on Green functions and poisson kernels for symmetric stable process, Math. Ann. 312, 465-501 (1998).

[9] H. Chen and L. Véron, Semilinear fractional elliptic equations involving measures, J. Differential Equations 257(5), 1457-1486 (2014).

[10] H. Chen and L. Véron, Semilinear fractional elliptic equations with gradient nonlinearity involving measures, J. Funct. Anal. 266(8), 5467-5492 (2014).

[11] H. Chen and L. Véron, Weakly and strongly singular solutions of semilinear fractional elliptic equations, Asymptotic Analysis 88, 165-184 (2014).

[12] E. Elshahed, A fractional calculus model in semilunar heart valvevibrations, International Mathematica symposium (2003).

[13] P. Felmer and A. Quaas, Fundamental solutions and Liouville type theorems for nonlinear integral operators, Advances in Mathematics 226, 2712-2738 (2011).

[14] P. Felmer and Y. Wang, Radial symmetry of positive solutions to equations involving the fractional laplacian, Comm. Cont. Math. 16, 1-24 (2014).

[15] A. Gmira and L. Véron, Boundary singularities of solutions of some nonlinear elliptic equations. Duke Math. J. 64(2), 271-324 (1991).

[16] Q. Guan and Z. Ma, Boundary problems for fractional Laplacians, Stochastics and Dynamics 5, 385-424 (2005).

[17] I. Herbst, Spectral Theory of the operator $\left(p^{2}+m^{2}\right)^{1 / 2}-Z e^{2} / r$, Commun. Math. Phys. 53, 285-294 (1977).

[18] E. Lieb and H. Yau, The Chandrasekhar theory of stellar collapse as the limit of quantum mechanics, Commun. Math. Phys. 112, 147-174 (1987).

[19] R. Magin, Fractional calculus in bioenginering 1, 2, 3, Critical Reviews in Biomedical Engineering 32, 1-104, 105-193, 194-377 (2004). 
[20] R. Magin, S. Boregowda, and C. Deodhar, Modeling of pulsating peripheral bioheat transf using fractional calculus and constructal theory, Journal of Design Nature 1, 18-33 (2007).

[21] R. Magin, X. Feng, and D. Baleanu, Fractional calculus in NMR, Magnetic Resonance Engineering 34, 16-23 (2009).

[22] R. Magin and M. Ovadia, Modeling the cardiac tissue electrode interface using fractional calculus, Journal of Vibration and Control 19, 1431-1442 (2009).

[23] M. Marcus and L. Véron, The boundary trace of positive solutions of semilinear elliptic equations: the subcritical case, Arch. Rat. Mech. Anal. 144, 201-231 (1998).

[24] M. Marcus and L. Véron, The boundary trace of positive solutions of semilinear elliptic equations: the supercritical case, J. Math. Pures Appl. 77, 481-524 (1998).

[25] M. Marcus and L. Véron, Removable singularities and boundary trace, J. Math. Pures Appl. 80, 879-900 (2000).

[26] M. Marcus and L. Véron, Capacitary estimates of positive solutions of semilinear elliptic equations with absorption, J. European Math. Soc. 6, 483-527 (2004).

[27] E. Di Nezza, G. Palatucci and E. Valdinoci, Hitchhiker's guide to the fractional Sobolev spaces, Bull. Sci. Math. 136, 521-573 (2012).

[28] X. Ros-Oton and J. Serra, The Dirichlet problem for the fractional laplacian: regularity up to the boundary, J. Math. Pures Appl. 101(3), 275-302 (2014).

[29] V. Tarasov and G. Zaslavsky, Fractional dynamics of systems with long-range interaction. Commun. Nonlinear Sci. Numer. Sim. 11, 885-889 (2006). 